\title{
RESEARCH
}

Open Access

\section{CD10 marks non-canonical PPARY- independent adipocyte maturation and browning potential of adipose-derived stem cells}

Smarajit Chakraborty ${ }^{1,2}$, Wee Kiat Ong ${ }^{1,3+}$, Winifred W. Y. Yau ${ }^{4 \dagger}$, Zhihong Zhou ${ }^{1}$, K. N. Bhanu Prakash ${ }^{5}$, Sue-Anne Toh ${ }^{6}$, Weiping Han ${ }^{7}$, Paul M. Yen ${ }^{4}$ and Shigeki Sugii ${ }^{1,2,4^{*}}$

\begin{abstract}
Background: Effective stem cell therapy is dependent on the stem cell quality that is determined by their differentiation potential, impairment of which leads to poor engraftment and survival into the target cells. However, limitations in our understanding and the lack of reliable markers that can predict their maturation efficacies have hindered the development of stem cells as an effective therapeutic strategy. Our previous study identified CD10, a pro-adipogenic, depot-specific prospective cell surface marker of human adipose-derived stem cells (ASCs). Here, we aim to determine if $\mathrm{CD} 10$ can be used as a prospective marker to predict mature adipocyte quality and play a direct role in adipocyte maturation.

Methods: We first generated 14 primary human subject-derived ASCs and stable immortalized CD10 knockdown and overexpression lines for 4 subjects by the lentiviral transduction system. To evaluate the role of CD10 in adipogenesis, the adipogenic potential of the human subject samples were scored against their respective CD10 transcript levels. Assessment of UCP1 expression levels was performed to correlate CD10 levels to the browning potential of mature ASCs. Quantitative polymerase chain reaction ( $(\mathrm{PCR})$ and Western blot analysis were performed to determine CD10-dependent regulation of various targets. Seahorse analysis of oxidative metabolism and lipolysis assay were studied. Lastly, as a proof-of-concept study, we used CD10 as a prospective marker for screening nuclear receptor ligands library.

(Continued on next page)
\end{abstract}

\footnotetext{
* Correspondence: shigekis@ibn.a-star.edu.sg

${ }^{+}$Wee Kiat Ong and Winifred W. Y. Yau contributed equally to this work.

${ }^{1}$ Fat Metabolism and Stem Cell Group, Singapore Bioimaging Consortium

(SBIC), Agency for Science, Technology and Research (A*STAR) Singapore, 11 Biopolis Way, Singapore 138667, Singapore

${ }^{2}$ Xenobiology Division, Institute of Bioengineering and Nanotechnology (IBN) Singapore, A*STAR, 31 Biopolis Way, Singapore 138669, Singapore

Full list of author information is available at the end of the article
}

C C The Author(s). 2021 Open Access This article is licensed under a Creative Commons Attribution 4.0 International License, which permits use, sharing, adaptation, distribution and reproduction in any medium or format, as long as you give appropriate credit to the original author(s) and the source, provide a link to the Creative Commons licence, and indicate if changes were made. The images or other third party material in this article are included in the article's Creative Commons licence, unless indicated otherwise in a credit line to the material. If material is not included in the article's Creative Commons licence and your intended use is not permitted by statutory regulation or exceeds the permitted use, you will need to obtain permission directly from the copyright holder. To view a copy of this licence, visit http://creativecommons.org/licenses/by/4.0/. The Creative Commons Public Domain Dedication waiver (http://creativecommons.org/publicdomain/zero/1.0/) applies to the data made available in this article, unless otherwise stated in a credit line to the data. 
(Continued from previous page)

Results: We identified intrinsic CD10 levels as a positive determinant of adipocyte maturation as well as browning potential of ASCs. Interestingly, CD10 regulates ASC's adipogenic maturation non-canonically by modulating endogenous lipolysis without affecting the classical peroxisome proliferator-activated receptor gamma (PPARY)dependent adipogenic pathways. Furthermore, our CD10-mediated screening analysis identified dexamethasone and retinoic acid as stimulator and inhibitor of adipogenesis, respectively, indicating CD10 as a useful biomarker for pro-adipogenic drug screening.

Conclusion: Overall, we establish CD10 as a functionally relevant ASC biomarker, which may be a prerequisite to identify high-quality cell populations for improving metabolic diseases.

Keywords: Biomarker, adipogenesis, Quality, adipose-derived mesenchymal stem cells, Adipocytes, beige, Adipocytes, non-canonical activation, Lipolytic pathways, Seahorse analysis, oxidative metabolism, Neprilysin, NEP, MME, Drug screening, nuclear receptor superfamily, Lipid droplets accumulation

\section{Background}

White adipose tissue (WAT) is primarily involved in storing lipids and secrete bioactive adipokine and cytokine dysfunction of which leads to reduced adipogenesis, metabolic syndrome, inflammation, insulin resistance, and type 2 diabetes [1-5]. Adipose-derived stem/stromal cells (ASCs) which constitute $\sim 1 \%$ of WAT are well defined as a mesenchymal stem cell type by the International Society for Cell and Gene Therapy (ISCT) based on their adherence, multipotency, and presence of selected markers [6]. By virtue of their differentiative and immunobiological properties, ASCs promise to be immunomodulators and gene delivery vehicles and possess a significant therapeutic impact in the field of regenerative medicine [7-9]. Several lines of evidence have shown pathophysiological differences between subcutaneous fat versus visceral fat in terms of interaction, secretion, insulin sensitivity, inflammation, circulation, maturation, metabolic, and lipid profiles [10-12]. Whether these differences originate from the stem cell populations only recently have started to be revealed.

Our group previously presented definitive cell surface markers and molecular signatures of ASCs derived from subcutaneous fat and visceral fat depots [6, 13]. Our high content screening assay of over 240 human cell surface markers identified CD10 (a.k.a. membrane metalloendopeptidase, MME) to be a subcutaneous fatspecific factor. We further showed that these markers can be used to enrich distinct populations of stem cells by their potential adipogenic capabilities; when sorted, $\mathrm{CD} 10^{\mathrm{hi}}$ ASCs differentiated into adipocytes better than $\mathrm{CD} 10^{\text {lo }}$ counterparts [6]. These results implicated potential use of CD10 as a prospective marker for high-quality adipogenesis and its potential direct role in the adipogenic process. However, it remained to be established if this stem cell marker can be used to indicate later phase adipocyte maturation capacities of ASCs across different human subject samples and if CD10 plays a direct role in terminal differentiation.
In contrast to WAT, brown adipose tissue (BAT) specializes in expending energy and generating heat through the specific function of uncoupling protein-1 (UCP1), which in turn leads to improved systemic glucose and lipid homeostasis $[14,15]$. Although BAT is abundant in rodents and infants but not in human adults, inducible, browning (also termed "beige" or "brite") adipocytes are discovered present inside subcutaneous WAT and resemble biological characteristics of BAT [16-18]. Thus, inducible characteristics of beige cells make them a promising therapeutic target for obesity treatment. There is no report of stem cell markers to our knowledge that indicate beiging/browning abilities of ASCs.

In this manuscript, we aim to determine if CD10 can be used as a marker to predict adipocyte maturation and browning potential of ASCs. We successfully delineate the impact of $\mathrm{CD} 10$ on adipogenic differentiation capacity by using human knockdown (KD) and overexpression (OE) lines. We report that CD10-driven adipocyte maturation of ASCs is independent of the classical PPAR $\gamma$-dependent adipogenic pathway and thus, is noncanonical. Further, we investigate if CD10 can be used as a biomarker to screen for ASCs with higher adipogenic capacities, which is a prerequisite to improve quality of fat cells important for preventing metabolic abnormalities.

\section{Materials and methods}

\section{Isolation and culture of ASCs}

WAT was isolated from the subcutaneous (abdominal region) depot from all the human volunteers undergoing bariatric surgery with approval from the Domain Specific Review Board at National Healthcare Group, Singapore. Information of the 12 donors is described in Supplementary Table S1. ASCs were isolated and enriched by serial passage culture of stromal vascular fractions (SVF), as described previously $[6,19]$. Immortalized ASCs were established from subject S29, with lentivirus using the pLVX-puro-Myc plasmid and Lenti-X HT Packaging 
System. The immortalized ASCs retained normal differentiation levels up to passage 30 (data not shown).

\section{Adipogenesis, browning, and AdipoRed staining}

Two days after reaching confluency, ASCs were induced with adipogenic cocktail containing $1 \mathrm{mM}$ dexamethasone (Sigma, USA), 0.5 mM IBMX (Sigma, USA), and $167 \mathrm{nM}$ insulin plus (Gibco, USA) $100 \mathrm{mM}$ indomethacin (Sigma, USA) as reported previously [6]. On D6, cells were switched to medium with $167 \mathrm{nM}$ insulin and $1 \mathrm{mM}$ dexamethasone and maintained until D12. The medium was changed every 3 days till D12. To induce browning, differentiated ASCs were treated with either $10 \mathrm{mM}$ Forskolin (FSK) (Sigma, USA) or DMSO control for $6 \mathrm{~h}$. For the AdipoRed staining of lipid droplets (LDs), AdipoRed ${ }^{\text {tw }}$ Assay Reagent (Lonza, USA) was used in a 96-well format according to the manufacturer's instructions with modifications. The cells were stained with the dye reagent for $30 \mathrm{~min}$ followed by $2 \mathrm{X}$ wash with HBSS. The nuclei of cells were then counterstained with Hoechst 33342 (Thermo Fisher Scientific, USA) for 15 min before proceeding for imaging.

\section{Oil Red 0 staining}

After adipogenesis, the cells were fixed in 3.7\% formaldehyde in PBS for $1 \mathrm{~h}$, washed with 60\% isopropanol, air-dried, and stained with Oil Red O solution (in 60\% isopropanol) as described previously [6]. Staining was extracted by isopropanol and its absorbance was measured at $500 \mathrm{~nm}$ by a spectrophotometer.

\section{Microscopy}

Imaging was performed by using a high-content automated fluorescence microscope system ImageXpress (Molecular Devices, USA) equipped with AgileOptix ${ }^{\mathrm{max}}$ technology. Images were obtained with a $\times 10$ objective lens in a $100 \mathrm{w}$ pillar diascopic illuminator with TE-C ELWD condenser. Software ImageJ was used to analyze the images. Two sets of images were taken corresponding to (i) AdipoRed ${ }^{\text {st }}$ reagent emission wavelength (536/ $40 \mathrm{~nm}$ ) upon $506 \mathrm{~nm}$ donor excitation and (ii) Hoechst 33342 emission wavelength $(447 / 60 \mathrm{~nm})$ upon $409 \mathrm{~nm}$ excitation.

\section{Quantification of lipid droplets}

A comprehensive automated MATLAB algorithm, which was adapted from our previously developed computational method [20], was used to count the nuclei number, measure the number of LDs normalized to the nuclei counts, and score the percentage of nuclei associated with lipids. Intensity, neighborhood, and shape features were used in the method to characterize the LDs and their association to nuclei that are used in the calculation of the ratio of total lipid intensity (total amount in pixels) to the corresponding nuclei intensity (total amount in pixels).

\section{Knockdown and overexpression of CD10}

Short hairpin RNA (shRNA) constructs were annealed, phosphorylated, ligated, and cloned into lentiviral pKAMU H1 vector backbone, using BamHI/EcoRI restriction sites. The shRNA construct was then transformed into Escherichia coli DH5 $\alpha$ cells. Positive clones were selected by sequencing the plasmid vector. Lentivirus was produced by co-transfecting human embryonic kidney 293 (HEK293T) cells with pKAMU containing either scrambled sequence (control) or CD10 shRNA, along with pMDL, pREV, and pVSVG. Media containing virus was collected after $48 \mathrm{~h}$, which was subsequently concentrated by ultracentrifugation. Immortalized ASCs were then infected with either control or CD10-shRNA lentiviral vectors at a multiplicity of infection (MOI) 10 . ASCs were incubated with puromycin for $48 \mathrm{~h}$ postinfection and kept for at least a week to select for successfully infected ASCs.

To study overexpression of CD10, the control vector (iRFP720-P2A) was constructed by cloning P2A, a porcine teschovirus-1-derived self-cleaving $2 \mathrm{~A}$ peptide, tandemly with image tracking reporter iRPF720 by $2 \mathrm{~A}$ technology [21]. The iRFP720 gene was amplified from piRFP720-N1 (Addgene plasmid \#45461) with a flanking 5 prime NheI site and 3 prime NotI sites including the full P2A sequence. The purified iRFP720 gene was then cloned into the multiple cloning sites (MCS) of expression lentivector pCDH-EF1-MCS-pA-PGK-copGFPT2A-Puro (Addgene plasmid \#CD550A-1). The overexpression vector (iRFP720-P2A-CD10) was designed in 2 steps. First, the CD10 gene was PCR amplified flanked by 5 prime NheI-SalI site and 3 prime Not I site and cloned into the MCS of vector pCDH-EF1-MCS-pAPGK-copGFP-T2A-Puro. Next, the iRFP720-P2A fragment containing the 5 prime NheI site and 3 prime SalI site was PCR amplified and cloned into the respective NheI-Sall site of the vector obtained from the first step. All the above-mentioned vectors were verified by further sequencing of the identified clones. These lentiviral vectors were infected to immortalized ASCs to establish stable lines.

\section{Quantitative real-time PCR}

Total RNA from the cultured cells was extracted using TRIzol reagent (Invitrogen, USA) and treated with DNase I to remove genomic DNA. cDNA conversion and analysis was done as previously described [6]. Relative mRNA levels were calculated and normalized to that of human ribosomal protein L27 (RPL27). The primers are listed in Supplementary Table S2. 


\section{Seahorse XF analyzer measurement for mitochondrial oxygen consumption rate (OCR)}

ASCs were seeded on XF-24-well culture microplates and allowed to differentiate as mentioned above. Differentiated ASCs were then treated with either $10 \mathrm{mM}$ Forskolin (FSK) or DMSO control for $6 \mathrm{~h}$. After treatment, oxygen consumption was measured using a microplate (type XF24) extracellular analyzer (Seahorse Bioscience, Billerica, MA, USA) as described in [22].

\section{Immunoblot analysis}

Cells were dissociated in RIPA buffer $(50 \mathrm{mM}$ Tris- $\mathrm{HCl}$, pH 8.0, 150 mM sodium chloride, $1 \%$ Triton X-100 (BioRad), $0.5 \%$ sodium deoxycholate (Sigma, USA), $2 \mathrm{mM}$ EGTA, 2 mM EDTA, 0.1\% sodium dodecyl sulfate, protease inhibitors (Sigma, USA), and phosphatase inhibitors (Sigma, USA); proteins were denatured by boiling in Laemmli sample buffer $(250 \mathrm{mM}$ Tris- $\mathrm{HCl}, \mathrm{pH} 7.4,2 \% \mathrm{v}$ : v sodium dodecyl sulfate, $25 \%$ v:v glycerol, $50 \mathrm{mM}$ DTT, $0.01 \%$ w:v bromophenol blue). Equal amount of proteins was resolved on sodium dodecyl sulfatenefapolyvinylidene difluoride membranes (Bio-Rad) using the TransBlot $^{\odot}$ Turbo $^{\text {Ta }}$ Transfer System (Bio-Rad). The following antibodies were used to detect the target proteins. CD10 (Leica Microsystmes), PPARG, phosphoAKT (Ser473), AKT, phospho-p70S6 (Thr389), phospho-70S6 (Thr470), phospho-AMPK (P-T172), AMPK, p62, LC3, ATF4, ATF6, phospho-HSL (Ser660), HSL, phosphor-ATGL (Ser406), ATGL, and COXIV. HRP-conjugated IgG (Santa Cruz) was used as the secondary antibody. The membranes were stained with Pierce $^{\mathrm{Tm}}$ ECL Plus (Thermo Fischer Scientific, USA) luminol/enhancer reagent and the X-ray film was developed by autoradiography. Antibodies used in this study are listed in Supplementary Table S3.

\section{Determination of lipolysis in matured ASCs}

Lipolysis was determined by estimating triglyceride breakdown and release of glycerol and non-esterified fatty acids (NEFA) from matured ASCs. Intracellular triglyceride levels were measured of fully differentiated ASCs by the Triglyceride Colorimetric Assay Kit (Caymen Chemical, USA). The amount of NEFA present in the pellet as well as released outside were estimated by the Free Fatty Acid Fluorometric Assay Kit (Caymen Chemical, USA). Glycerol release was measured by Glycerol Colorimetric Assay Kit (Caymen Chemical, USA). All the results were normalized per milligram of protein present.

\section{Nuclear ligand screening}

Screening was carried out using Screen-Well nuclear receptor ligand library (Enzo BML-2802) with 76 synthetic ligands $(10 \mu \mathrm{M})$ of nuclear receptors (AR, CAR, ER, GR,
FXR, LXR, PPAR, PXR, RAR, RXR, VDR). The assays were performed by immunofluorescence study using ImageXpress high content screening system and flow cytometry analysis with BD LSR Fortessa on ASCs from $\mathrm{S} 13$ that had been incubated with $10 \mu \mathrm{M}$ of the respective ligands for $\sim 68 \mathrm{~h}$. The screening was done by using high-throughput flow cytometry and image-based high content analyses (Supplementary Table S4). Selected candidate ligands of interest from the first screening were further tested by individual flow cytometry analysis (Fig. S4). Positive hits were applied on ASCs from S23 and verified by flow cytometry analysis.

\section{Flow cytometry}

ASCs were trypsinized and a total of $2 \times 10^{5}$ cells were suspended in $0.2 \mathrm{ml}$ staining buffer (DMEM without phenol red with $2 \%$ FBS) (Thermo Fisher Scientific, USA) for immunostaining. The cells were incubated for 30 min on ice, with a FITC-conjugated antibody against CD10 (BD Biosciences, USA). The stained cells were washed and suspended in sorting buffer (PBS with 0.5\% BSA and 2 mM EDTA) before analysis by flow cytometry (LSR FORTESSA, BD Biosciences).

\section{Statistical analysis}

Results were represented as means \pm SEM. Student's $t$ test was used to determine differences in means between two groups. The $p$ value was calculated using ANOVA for multiple comparisons with corrections and $p<0.05$ being considered as significant. Correlation coefficient $(R)$ value was calculated from the eq. $\left[R^{2}=\backslash\right.$ frac $\{\mid \operatorname{sum}\{(\mathrm{x}-$ $\left.\left.\left.\mathrm{m}_{-} \mathrm{x}\right)\left(\mathrm{y}-\mathrm{m} \_\mathrm{y}\right)\right\}\right\}\left\{\mid \operatorname{sqrt}\left\{\left|\operatorname{sum}\left\{\left(\mathrm{x}-\mathrm{m} \_\mathrm{x}\right)^{\wedge} 2\right\}\right| \operatorname{sum}\left\{\left(\mathrm{y}-\mathrm{m} \_\mathrm{y}\right)^{\wedge} 2\right\}\right\}\right\} ;$ $\mathrm{m}$, mean; $\mathrm{x}$, relative $C D 10$ level; $\mathrm{y}=$ relative $\mathrm{LDs}$ level].

\section{Results}

Intrinsic levels of CD10 determine the adipogenic maturation potential of ASCs

Based on our previous finding, we postulated that intrinsic levels of CD10 might positively predict the potential of ASC maturation into adipocytes [6]. We investigated human subjects-derived ASCs isolated from the subcutaneous depot from 12 donors within low passages to avoid possible cellular variation. These ASCs were subjected to the standard in vitro adipogenic cocktail and accumulation of LDs was quantified. To evaluate expression changes of $C D 10$, quantitative real-time PCR (qRTPCR) was performed in non-differentiated (D0; basal) and mature (D12) ASC populations. ASCs with higher basal CD10 levels (S49) showed robust adipogenesis whereas the lowest basal CD10 expressing ASC (S60) accumulated minimum LDs as quantified by the AdipoRed assay (Fig. 1a-c). All the 12 subjects showed moderate increase in $C D 10$ expression from D0 to D12 during the course of differentiation (Fig. 1b), in agreement to our 

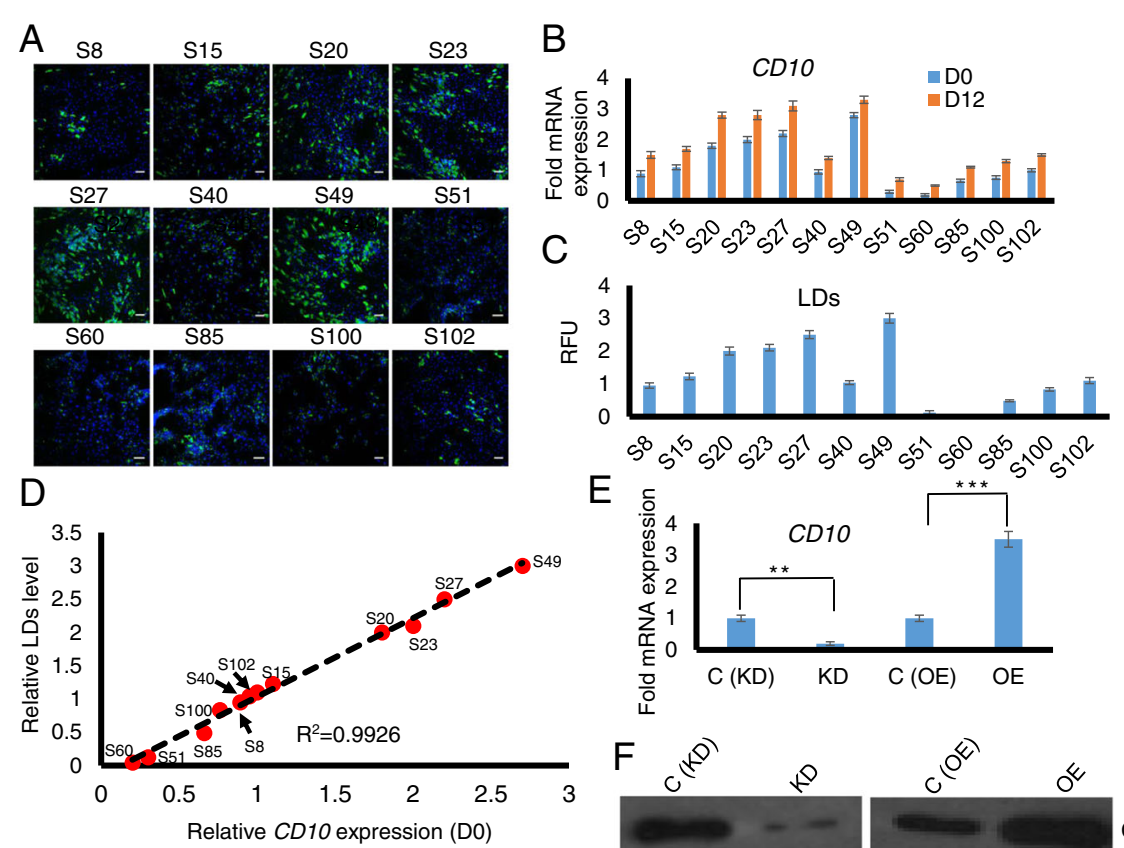

$\mathrm{E}$
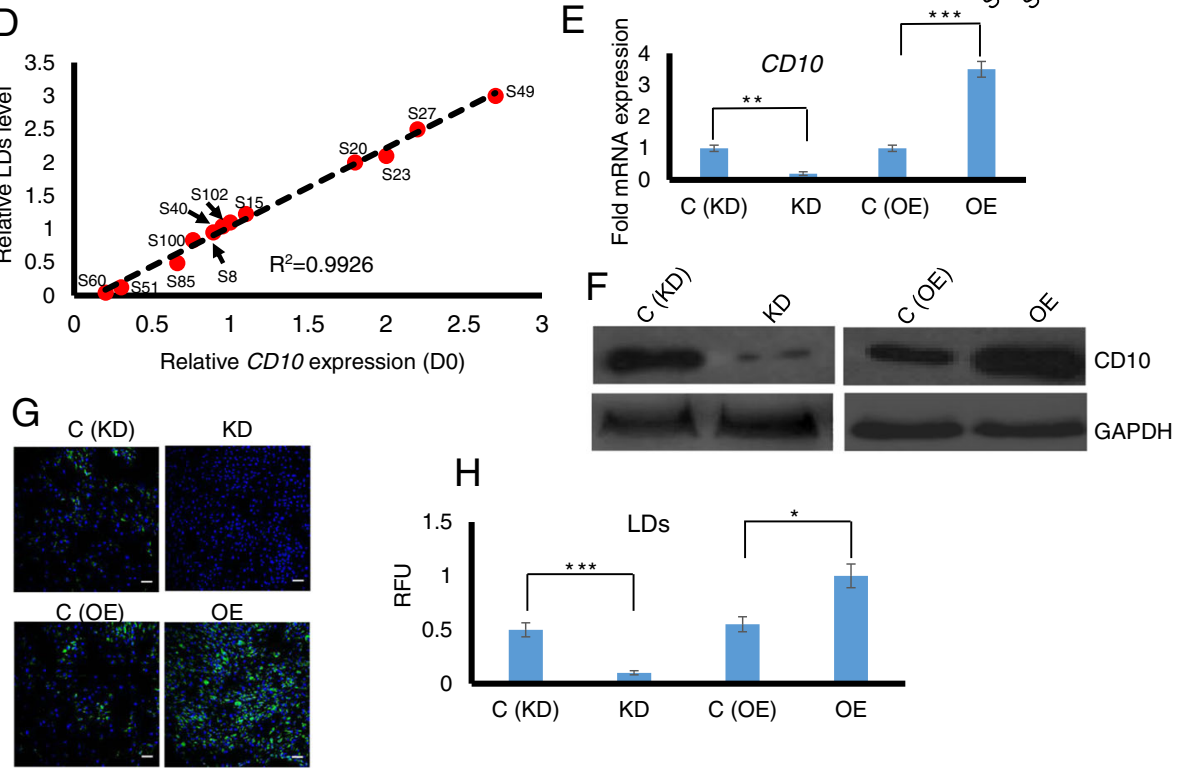

$\mathrm{H}$

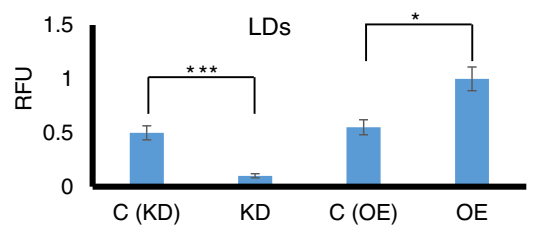

Fig. 1 Intrinsic levels of CD10 positively correspond to lipid accumulation. a Representative merged images of AdipoRed staining of lipids (in green) and Hoechst 33342 staining of nuclei (in blue) across differentiated ASCs from 12 subjects (between passage 6 and 8) are shown. The scale bar represents $100 \mu \mathrm{m}$. b CD10 levels at D0 (basal undifferentiated) and at D12 (differentiated) of ASCs from the 12 subjects are shown based on GRT-PCR analysis. Fold expression changes of CD10 are compared to the S8. Each value is the mean \pm SEM from three independent wells for qRT-PCR of each population performed in triplicates. c The LDs are quantified in the differentiated ASCs of the 12 subjects by using a MATLAB algorithm as a read-out for adipogenesis levels. Each value is the mean \pm SEM $(n=6)$. $\mathbf{d}$ Correlation of CD10 levels at D0 and lipids at D12 post-differentiation are shown. CD10 levels are highly correlated with the lipids accumulated upon differentiation (Correlation coefficient $R=$ 0.9962). e The relative mRNA expression levels of CD10 by qRT-PCR in CD10 knockdown (KD), CD10 overexpression (OE) compared with their respective controls $C(K D)$ and $C(\mathrm{OE})$ ASCs are shown normalized to RPL27. Fold expression changes are compared to the $C$ (KD) level. Each value is the mean \pm SEM from three independent replicates. $\mathbf{f}$ Western blot analysis shows the protein expression of CD10 in CD10 KD and CD10 OE ASCs compared to the respective controls. GAPDH was used as the loading control. $\mathbf{g}$ Representative merged images of AdipoRed staining of lipids (in green) and Hoechst 33342 staining of nuclei (in blue) of CD10 KD, CD10 OE and the control ASCs lines are shown. The scale bar represents $100 \mu \mathrm{m}$. h Quantification of the lipid content was done by using a MATLAB algorithm. CD10 OE and CD10 KD ASC populations showed respective increase and decrease in lipids compared to the controls. Each value is the mean \pm SEM for each population performed in five-independent batches. All statistical analysis was performed using Student's paired $t$ test, ${ }^{* * *} p<0.001,{ }^{* *} p<0.01,{ }^{*} p<0.05$

previous report [6]. The CD10 levels at D12 are consistent with those at D0 relative to individual subjects. Near-complete correlation (Pearson's $R^{2}=0.9926$ ) was observed between the basal CD10 levels (at D0) and LDs accumulation (at D12), indicating the intrinsic CD10 level as a strong predictor of mature adipocyte quality (Fig. 1d). Overall, our data highlights functional heterogeneity within subject-derived ASCs isolated from the same subcutaneous fat depot and suggests that high baseline CD10 is predictive of good adipogenic potentials in ASCs.

\section{CD10 directly impacts adipocyte maturation of ASCs}

To study the functional relevance of CD10 in regulating adipogenic maturation, we created stable knockdown (KD) and overexpression (OE) lines. Immortalized ASCs were stably transduced with CD10 shRNA or empty vector (control) to obtain CD10 KD and control cell 
populations. On the other hand, ASCs were transduced with CD10 overexpression or vector control as described in the "Materials and methods" section to obtain the CD10 OE and control cell lines. To examine the extent of overexpression and knockdown by the lentiviral transduction system, we investigated the expression of CD10 at both RNA and protein levels. The qRT-PCR analysis showed about 5-fold decrease in CD10 expression in the KD cells and 3.5-fold increase in OE cells compared to the respective controls (Fig. 1e). Changes in the protein expression were also confirmed by Western blot by using CD10-specific antibody (Fig. 1f). Next, we subjected the ASC lines into an in vitro standard adipogenic differentiation cocktail as described in the "Materials and methods" section. AdipoRed, a specific fluorescence dye for neutral lipids, was used to stain the LDs, and quantification was done by an automated algorithm based on the previously developed protocol (see the "Materials and methods" section) [20]. KD of CD10 significantly reduced LD accumulation upon differentiation, while CD10 OE enhanced LD signals, suggesting a positive correlation between basal CD10 levels and adipocyte maturation (Fig. 1g, h). In addition, we generated knockdown cell lines of 3 other subjects to further validate CD10-dependent adipocyte maturation by using a different LD-specific dye, Oil Red O. As expected, knockdown cells expressing low $C D 10$ levels as determined from the qRT-PCR analysis (Fig. S1A - S1C) exhibited significantly reduced LDs compared to the controls (Fig. S1D and Fig. S1E). Collectively, we conclude that CD10 directly and positively regulates adipocyte maturation of human ASCs.

\section{CD10-rich ASCs show increased beige phenotype upon stimulation}

We next tested if CD10 levels also predict the browning potential of mature ASCs. ASCs were differentiated with the standard adipogenic differentiation cocktail for 12 days and then treated with Forskolin (FSK) for $6 \mathrm{~h}$ to induce browning. Browning assessment from UCP1 expression and adipogenesis from LDs quantification were performed before and after the FSK treatment. Induced browning as evidenced by $U C P 1$ induction resulted in decreased lipid accumulation as quantified from the AdipoRed staining of LDs (Fig. 2a-c). Importantly, cells with higher intrinsic CD10 levels showed increased UCP1 upregulation compared to those with low CD10 levels (Fig. 2a, b). UPC1 upregulation showed good correlation to intrinsic CD10 levels (Pearson's $R^{2}=0.8068$ ) (Fig. 2d). Next, we compared if the reduction in LD accumulation as a consequence of induced browning is related to intrinsic CD10 levels. We observed moderate correlation (Pearson's $R^{2}=0.6440$ ) between lipid reduction and CD10 levels indicating CD10 as a potential marker of browning potential of ASCs (Fig. 2e). To investigate a direct effect of CD10 on browning potential of ASCs, we next compared UCP1 expression of CD10 OE and KD ASCs. As expected, CD10 OE ASCs showed the highest $U C P 1$ induction and maximum reduction in LDs upon browning treatment, whereas CD10 KD ASCs showed no UCP1 induction and no LDs reduction (Fig. $2 \mathrm{f}-\mathrm{h}$ ). Overall, our results establish CD10 as an additional functional determinant of browning potential of ASCs.

\section{CD10-induced adipocyte maturation of ASCs is non- canonical}

To elucidate the function of CD10, we first measured the expression levels of key adipogenic transcriptional factor, peroxisome proliferator-activated receptor gamma (PPARG), and specific late adipogenic gene, Fatty acid-binding protein 4 (FABP4; a.k.a. aP2) by qRT-PCR. Interestingly, PPARG and FABP4 levels across all 12 subjects remain unchanged as also observed in the differentiated CD10 KD or CD10 OE lines, which did not show significant changes compared to the respective controls (Fig. S2A - S2C). These results indicate that CD10-induced lipid accumulation is not via the classical adipogenic pathway mediated by the master regulator PPARY. We next investigated whether CD10 regulates other signaling pathways involved in adipocyte differentiation by examining the activation of specific targets such as AKT (PI3K/Akt pathway), p70S6 (mTOR pathway), 5' AMP-activated protein kinase (AMPK; AMPK pathway), p62 and LC3 (autophagy pathway), and Activating Transcription Factor 4 and 6 (ATF4 and ATF6; endoplasmic reticulum stress pathway). None of their players exhibited significant changes in their expression of both total protein and phosphorylation levels as evidenced by Western blot analysis (Fig. S2D), suggesting that CD10 acts independent of any of these pathways. These results implicate a more direct role of CD10 in regulating lipid formation of mature ASCs without going through the canonical adipogenic pathways.

\section{CD10 promotes lipid accumulation of mature ASCs by inhibiting lipolysis}

We reasoned that CD10 may be directly involved in de novo lipogenesis or lipolysis to regulate lipid accumulation in differentiated ASCs. We did not observe any significant changes in the expression level of fatty acid synthase (FAS) or phosphorylation of acetyl-CoA Carboxylase (ACC) in the CD10 KD or CD10 OE lines compared to the controls, suggesting non-involvement of CD10 in de novo lipogenesis (data not shown). Next, we investigated if CD10 regulates lipolysis by measuring the activation of key enzymes involved in intracellular lipid catabolism including hormone-sensitive lipase (HSL) 

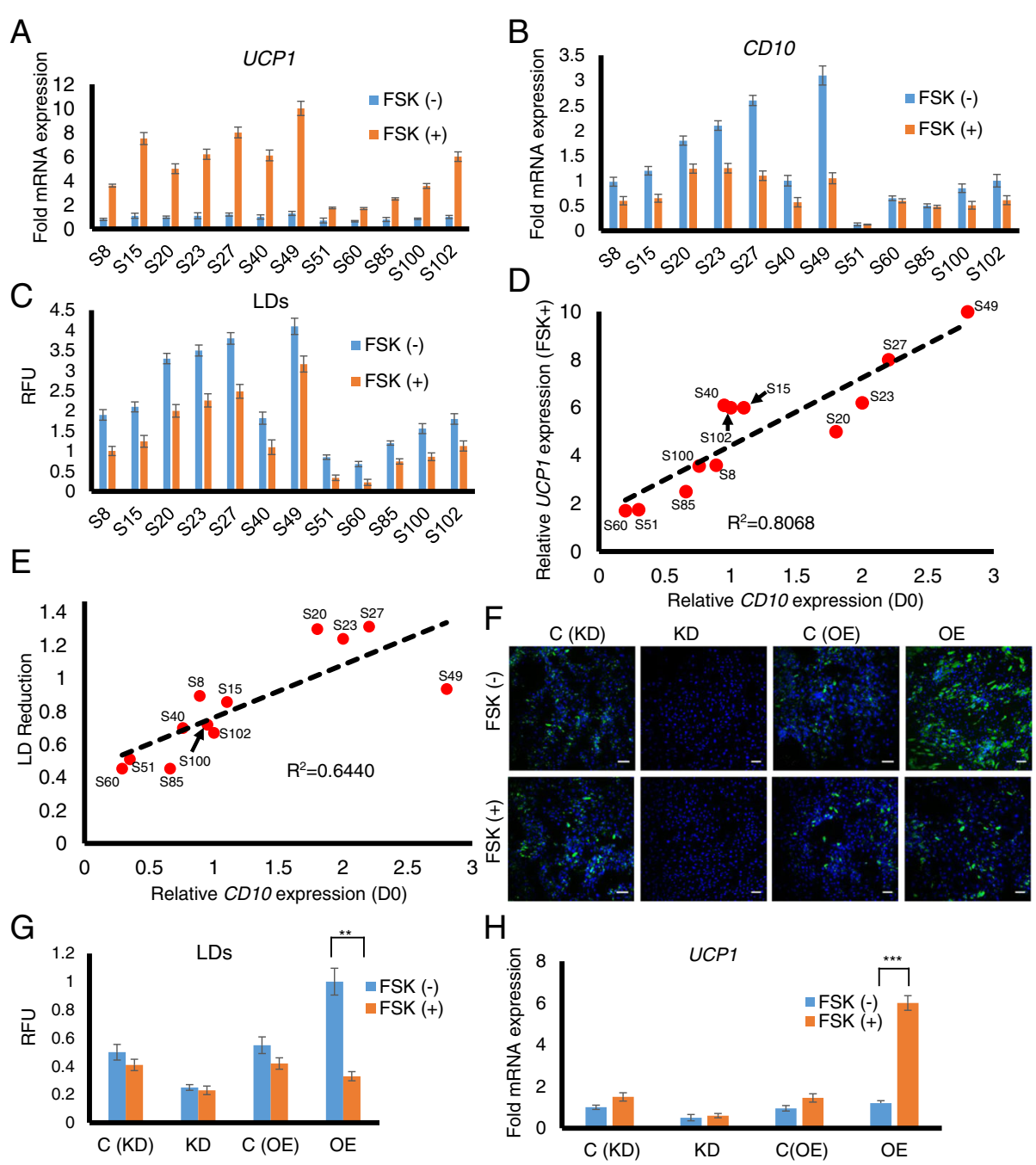

$\mathrm{H}$

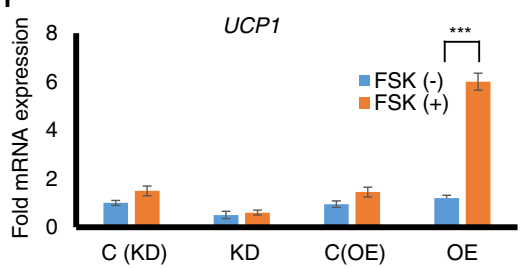

Fig. 2 High CD10 expressing ASCs show increased browning capacity. a Relative mRNA levels of UCP1 are quantified before and after FSK treatment by qRT-PCR normalized to RPL27. Fold expression changes are compared to the subject S8 without FSK treatment. Each value is the mean \pm SEM from three independent replicates. $\mathbf{b}$ Relative mRNA levels of CD10 are quantified at D0 and D12 by qRT-PCR normalized to RPL27. Fold expression changes are compared to the subject S102 (D0). Each value is the mean \pm SEM from three independent replicates. c The LDs are quantified by using MATLAB analysis before and after FSK treatment. $\mathbf{d}$ Correlation of CD10 levels with UCP1 expression are shown with the Pearson coefficient $R^{2}$ value. Good correlation is observed between UCP1 induction and CD10 levels $(R=0.8610)$. e Correlation between intrinsic CD10 levels with reduction in LDs following browning are shown with the Pearson coefficient $R^{2}$ value. $\mathbf{f}$ Representative merged images of AdipoRed staining of lipids (in green) and Hoechst 33342 staining of nuclei (in blue) in CD10 knockdown (KD) and overexpression (OE) cells with controls with (out) FSK treatment. The scale bar represents $100 \mu \mathrm{m}$. $\mathbf{g}$ The LDs are quantified by using MATLAB analysis as described in Experimental Procedures. The values are normalized to CD10 OE ASCs. Each value is the mean \pm SEM from three independent replicates. $\mathbf{h}$ Relative mRNA levels of browning marker gene UCP1 are quantified by qRT-PCR normalized to RPL27. Fold expression changes are compared to CD10 OE cells before FSK treatment. Each value is the mean \pm SEM from three independent replicates. All statistical significance was assessed by using Student's paired t-test: ${ }^{* *} p<0.001,{ }^{* *} p<0.01,{ }^{*} p<0.05$

and adipose triglyceride lipase (ATGL) of differentiated ASCs. Compared to the control cells, CD10 KD cells showed a significant increase in the phosphorylation of both HSL and ATGL, whereas CD10 OE cells exhibited reduced activation (Fig. 3a-f). Our data suggest that CD10 mediates lipid accumulation by suppressing the activation of HSL and ATGL during adipogenic differentiation of ASCs. Next, we postulated whether ATGL and HSL activation in mature CD10 KD cells results in heightened mitochondrial respiration/ $\beta$-oxidation, which is caused by excessive breakdown of triglycerides (TGs) from LDs. Indeed, the Seahorse XF Cell Mito Stress assay recorded increased basal respiration, ATP production, and further enhanced maximal mitochondrial respiration, accounting for the result of higher endogenous lipolysis in mature CD10 KD cells (Fig. 3g). Increased expression of Cytochrome c Oxidase Subunit IV (COXIV), an essential protein for mitochondrial 


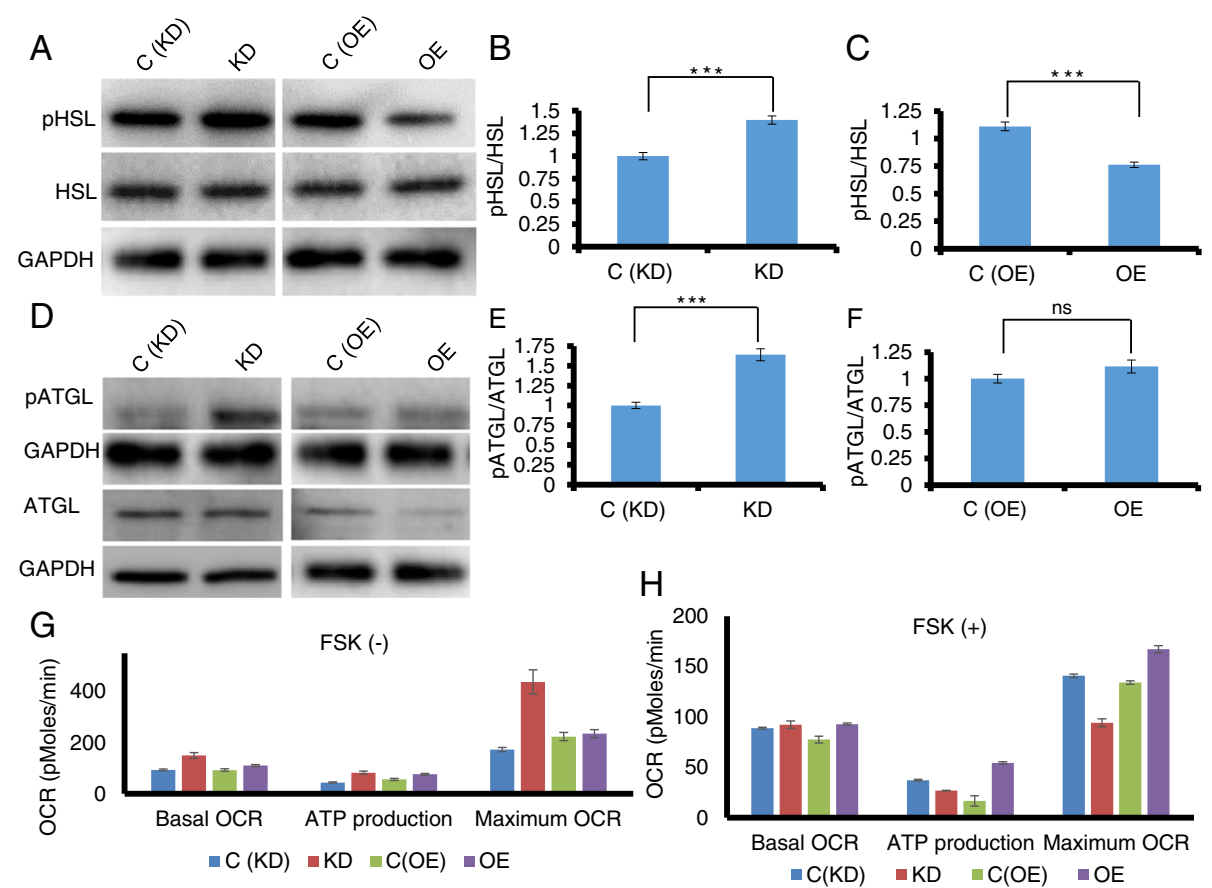

C
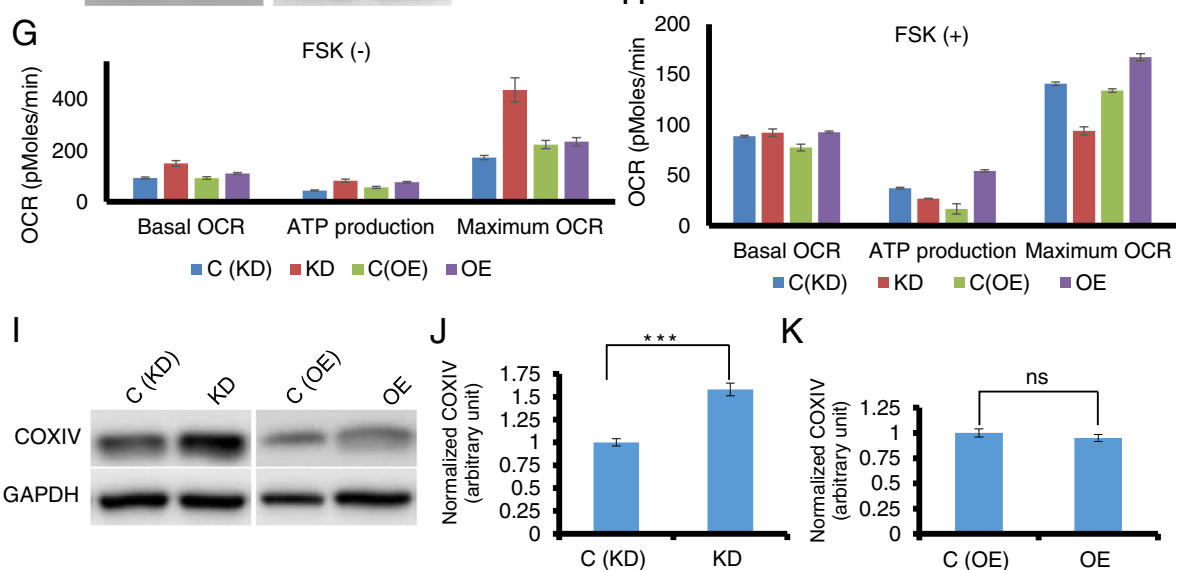

$\mathrm{K}$

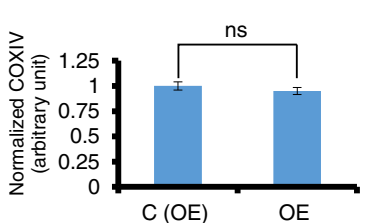

Fig. 3 CD10 inhibits endogenous lipolysis resulting in higher lipid accumulation in mature ASCs. Western blot analysis shows detection of a phosphorylated form of HSL and $\mathbf{d}$ ATGL using antibodies against phospho-HSL (P-S660), total HSL, phospho-ATGL (P-S406), and total ATGL in CD10 KD, OE, and control cells. Quantification of HSL phosphorylation are shown in $\mathbf{b}$ CD10 KD and c CD10 OE cells. Similar phosphorylation levels are shown for ATGL in e CD10 KD and $\mathbf{f}$ CD10 OE cells. CD10-mediated alteration on mitochondrial function expressed as Oxygen consumption rate (OCR; in pMoles/min) are shown in mature ASCs $\mathbf{g}$ before or $\mathbf{h}$ after FSK treatment. Western blot analysis showing the $\mathbf{i}$ expression of COXIV and its relative quantification between $\mathbf{j}$ CD10 KD and $\mathbf{k}$ OE cells compared to the controls. For all the blots, GAPDH was used as internal control, and statistical analysis was performed using Student's paired $t$ test $(n=3),{ }^{* * *} p<0.001,{ }^{*} p<0.05$, ns denotes non-significant, $p>0.05$

respiration, further validates heightened mitochondrial activity of CD10 KD cells (Fig. 3i-k). Next, we set out to quantify CD10-regulated intracellular lipolysis by comparing the amount of TGs accumulation and resultant release of free fatty acids (FAs) and glycerol between $\mathrm{CD} 10 \mathrm{KD}, \mathrm{OE}$, and control cells. In agreement with our finding, CD10 OE cells showed the highest accumulation of TGs and minimal release of FAs and glycerol into the media (Fig. S3). CD10 KD cells displayed lower triglyceride accumulation and maximum release of FAs and glycerol, conforming accelerated lipolysis (Fig. S3). Taken together, CD10 exerts a direct role in lipid accumulation during adipogenic maturation of ASCs by regulating intracellular lipolysis. As we observed that stem cell CD10 levels also positively mark the browning potential of ASCs (Fig. S1I), we reasoned that increased adipocyte browning may correspond to higher mitochondrial function in CD10 OE cells. Basal and maximum mitochondrial respiration rates along with ATP production were significantly raised in CD10 OE cells after induction with FSK, validating a strong association between adipocyte browning and mitochondrial activity (Fig. 3h). Collectively, while CD10 directly regulates lipolysis and mitochondrial metabolism, it also impacts the ability of mature ASCs to undergo browning and accompanied mitochondrial activities in response to the stimuli.

\section{Nuclear receptor ligand screening with CD10 as a} biomarker identifies dexamethasone as a promoter and retinoic acid as an inhibitor of adipogenesis

Our results indicate that $\mathrm{CD} 10$ can be a useful biomarker for pro-adipogenic capacities in drug screening. As a proof-of-concept, a mini-screening study using high content imaging of ASCs treated with nuclear receptor 
ligand library (with 76 ligands) was performed as described in the "Materials and methods" section. Nuclear receptor ligands were chosen because many members of this superfamily have been shown to play definitive roles in the processes of adipocyte development [23-25]. The top hit that increased CD10 expression was dexamethasone, one of the standard ingredients of adipogenic cocktail. In contrast, retinoic acid, a metabolite of vitamin A (retinol), showed maximum inhibition (Fig. S4). To study the pro-adipogenic potential of dexamethasone in detail, we divided the adipogenic differentiation processes into 3 schemes (Fig. 4a) and examined them on low (CD10 KD), high (CD10 OE), and intermediate CD10-expressing (S40 and S8) ASCs. In (i), ASCs were pre-treated with dexamethasone for $\sim 48 \mathrm{~h}$ and maintained throughout the adipogenic process (D-2 to D12); in (ii), dexamethasone was added during the adipogenic treatment without pre-treatment (D0 to D12); and in (iii), no dexamethasone was added at all. Dexamethasone was necessary for adipogenesis to take place as negligible LDs accumulated in the absence of dexamethasone in all the lines including CD10 OE ASCs (Fig. 4b, c). Pre-treatment with dexamethasone also showed a significant increase of $C D 10$ expression, which in turn resulted in heightened lipid accumulation in all the cell lines except for the CD10 KD cells (Fig. 4b-d). These findings suggest that while CD10 or dexamethasone alone is not sufficient for inducing adipogenesis, CD10 and dexamethasone are important and complementary in enhancing adipogenesis. In the absence of either $\mathrm{CD} 10$ or dexamethasone, the adipogenic process is significantly inhibited.

On the other hand, pre-treatment and maintenance with retinoic acid in the differentiation cocktail completely abrogated lipid accumulation and significantly decreased CD10 expression compared to the DMSO control (Fig. 4e-g), which is in agreement with our previous study [13]. CD10 OE could not relieve the retinoic acid-mediated adipogenic defects. Taken together, our screening using CD10 as a marker could successfully identify drugs that profoundly affect adipogenesis, which further validates $\mathrm{CD} 10$ as a prospective marker of mature adipocyte quality.

\section{Discussion}

Studying adipogenesis of ASCs is important for both pathophysiological understanding and quality control aspects of stem cell therapy. It is now evident that adipogenic capability of ASCs depends upon the quality of their originated adipose tissue $[6,12,26]$. There is substantial evidence that imbalance in lipid homeostasis is a major etiological factor resulting in lipo-toxicity and cell death and contributes towards chronic diseases such as insulin resistance and diabetes, cardiovascular disease, immunological and inflammatory disorders, and some types of cancer [27-32]. Therefore, understanding changes in lipid metabolism during stem cell differentiation may offer new insights that could lead to the development of novel, cell-based diagnostic and therapeutic strategies. In general, the ability of ASCs to fully differentiate into mature adipocytes is correlated with the quality of fat tissue that affects metabolic outcomes $[6,9,13]$. Thus, compounds that promote adipogenesis of ASCs may be good candidates for ultimately improving such metabolic diseases. Since ASCs are mesenchymal stem cells that possess trilineage differentiation capacities, the adipogenesis assay is one of the common methods to assess their stem cell potency. However, it typically takes about 2 weeks to perform adipocyte differentiation assay, which may be too timeconsuming and laborious for high throughput screening and routine stem cell differentiation protocols. In this paper, we report that CD10 offers a convenient marker that predicts how well ASCs differentiate into mature adipocytes (Fig. 1). Analysis of ASCs with CD10 takes 2 or less days, versus approximately 14 days for the adipocyte differentiation assay. As a proof-of-concept drug screening study, we found that dexamethasone, which significantly induces CD10 expression, enhances adipogenesis substantially (Fig. 4). In contrast, treatment with retinoic acid, which significantly reduces CD10 expression and inhibits adipogenesis, is consistent with our previous report [13]. These results indicate the suitability of $\mathrm{CD} 10$ as a prospective marker to investigate the quality of adipocytes with relative ease and speed.

We also found that CD10 directly affects lipid accumulation during adipogenesis (Fig. 1). Stable KD of CD10 reduces whereas its OE increases the accumulation of LDs. Investigation of ASCs from human subjects also confirmed tight correlation between basal CD10 levels and later adipogenesis degrees. These results suggest that it is possible to assess the quality of fat from individuals by examining the levels of intrinsic CD10 as a prospective marker in ASCs. There has been increasing interest to employ ASCs clinically for a number of metabolic disease conditions [8]. In addition to fat tissue for transplantation, ASCs now are actively investigated to improve metabolic outcomes in chronic diseases such as obesity and diabetes [33, 34]. In the future, it may be possible to use CD10 to screen and isolate a population of healthy and competent ASCs that are suitable for transplantation and capable of forming good quality fat tissue that can improve metabolic outcomes.

CD10 is a $100-k D a$ glycoprotein with neutral endopeptidase activity (NEP) which metabolizes bioactive proteins involved in various physiological processes and regulates their extracellular availability for receptors in the kidney and circulatory and nervous system [35, 36]. CD10 can directly mediate intracellular signaling event 


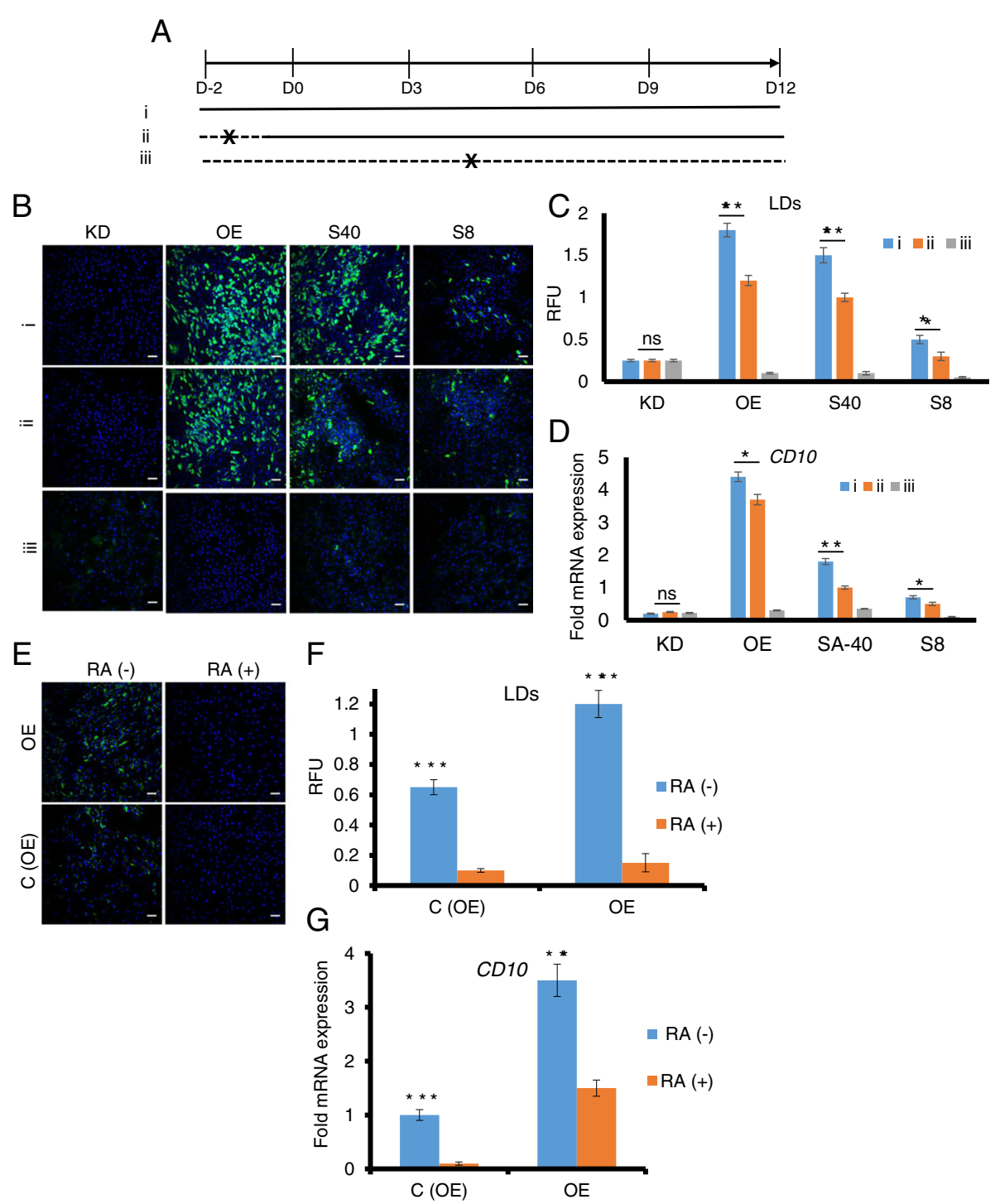

Fig. 4 Drug screening with CD10 as a marker identified dexamethasone and retinoic acid, which exhibit stimulatory and inhibitory effects on adipogenesis, respectively. a Dexamethasone $(10 \mu \mathrm{M}$ ) was added under three conditions such as (i) D-2 to D12 (pre-induction and adipogenic stimulation), (ii) D0 to D12 (only adipogenic stimulation), and (iii) no addition. The presence and absence of dexamethasone are shown as solid and dotted lines, respectively. b Representative merged images of AdipoRed staining of lipids (in green) and Hoechst 33342 staining of nuclei (in blue) are shown in CD10 KD, CD10 OE, S40, and S8 ASCs. The scale bar represents $100 \mu \mathrm{m}$. c The LDs are quantified as mentioned earlier as a read-out of adipogenesis levels. Statistical analysis was performed using Student's paired $t$ test $(n=3)$ between conditions (i) and (ii), ** $p<0.01$, ${ }^{*} p<0.05$, non-significant (ns) represents $p$ value $>0.05$. $\mathbf{d}$ Relative mRNA levels of CD10 are quantified under the indicated conditions of dexamethasone addition normalized to RPL27. Fold expression changes are compared to the subject S40 (ii). Pre-treatment with dexamethasone significantly increased CD10 expression and subsequently lipid accumulation except for the CD10 KD line. Statistical analysis was performed using Student's paired $t$ test $(n=3)$ between condition (i) and (ii), ${ }^{* *} p<0.01,{ }^{*} p<0.05$, non-significant (ns) represents $p$ value $>0.05$. Each value is the mean \pm SEM from three independent replicates. e Representative merged images of AdipoRed staining of lipids (in green) and Hoechst 33342 staining of nuclei (in blue) are compared between CD10 OE and control ASCs to see the effect of retinoic acid (RA) on adipogenesis. Equivalent amount of either DMSO or RA $(10 \mu \mathrm{M})$ was added. The scale bar represents $100 \mu \mathrm{m}$. $\mathbf{f}$ LDs was quantified based on MATLAB programming. Addition of RA significantly reduced LDs accumulation. $\mathbf{g}$ Relative mRNA levels of CD10 in presence or absence of RA were quantified. Fold expression changes are compared to C (OE) values. RA significantly reduced CD10 expression, and CD10 OE does not rescue RA-mediated adipogenic impairment. Each value is the mean \pm SEM from three independent replicates. Significance was assessed by using Student's paired $t$ test: ${ }^{* *} p<0.001$ 
via glycosylphosphatidylinositol (GPI) complexes [37]. We found that CD10-dependent lipid accumulation was non-canonical, independent of classical adipogenic pathways that are mediated through the master adipogenic regulator, PPAR $\gamma$ (Fig. S2). It is believed that almost all adipogenic processes, including lipid accumulation, in mature adipocytes are mediated by PPAR $\gamma[38,39]$. Natriuretic peptides (NP) and major hormones stimulating catabolic pathway in humans, and have been reported to be the direct substrates of CD10 [29, 40, 41]. In adipocytes, atrial NP (ANP) and brain type NP (BNP) stimulated NP receptor $A$ and induce a cGMP dependent pathway [42, 43]. Increased intracellular cGMP levels activate cGMPdependent protein kinase $G$, which in turn, induces lipolysis through phosphorylation of HSL [42]. After phosphorylation, HSL is translocated into the LDs and acts together with ATGL, accelerating the lipolytic process (Fig. S5). Together, ATGL and HSL are responsible for about 95\% of TAG hydrolysis [43-47]. We observed upregulation of both HSL and ATGL activities in CD10 KD cells, implying that CD10 mediates suppression of endogenous lipolysis, and in turn, promotes lipid accumulation in mature ASCs (Fig. 3 and Fig. S3, S5). This CD10-induced inhibition of lipolysis may act as an adaptive protective mechanism to minimize FA release and its deleterious consequences on systemic metabolism in obese and insulin-resistant subjects [48-50]. Previously, the lipolytic process was believed to depend primarily upon PPAR $\gamma$-mediated pathways in mature adipocytes [51-53]. In contrast, our current finding demonstrates a heretofore unrecognized role of a PPAR $\gamma$-independent factor, CD10, to be involved in lipid accumulation and maturation of adipocytes (Fig. S5).

The inducible browning (beige) adipocytes differ in origin and possess a distinct molecular signature from classical brown adipocytes; however, they both share similar characteristics such as high mitochondria content and UCP1 expression upon activation [54]. Several studies have shown that the same stimuli that increased classical brown fat activity in mice also resulted induced browning [55-58]. These studies suggest that they may be reproducible in man and indicate that inducing or transplanting brown/beige fat may be potential strategies for treating obesity and its associated disorders. In this study, we have found that basal CD10 level is correlated with the browning degree of ASCs, establishing CD10 as the prospective marker to indicate browning capability of stem cells (Fig. 2). It is presumed that the higher lipid accumulation found in CD10-high mature ASCs also is a prerequisite for enhanced browning capability of these cells, which upon appropriate stimulation leads to higher mitochondrial oxidative respiration during browning (Fig. 3H).

\section{Conclusions}

Our data demonstrate that CD10 regulates a novel, noncanonical adipocyte maturation process, which is essential for ASC maturation into high-quality WAT and browning. Thus, CD10 expression in ASCs may play an important role in preventing the development of obesity and other metabolic complications. Moreover, as a functional biomarker, CD10 promises to have clinical relevance and diverse applications for drug screening, differentiation assessment, and therapeutics related to obesity and diabetes.

\section{Supplementary Information}

The online version contains supplementary material available at https://doi. org/10.1186/s13287-021-02179-y.

\section{Additional file 1.}

\section{Abbreviations}

ASCs: Adipose-derived stem cells; PPARy: Peroxisome proliferator-activated receptor gamma; WAT: White adipose tissue; BAT: Brown adipose tissue; UCP1: Uncoupling protein-1; LDs: Lipid droplets; HSL: Hormone-sensitive lipase; ATGL: Adipose triglyceride lipase; TGs: Triglycerides; FAs: Free fatty acids; NEP: Neutral endopeptides; NP: Natriuretic peptides; ANP: Atrial NP BNP: Brain type NP; NPRA: NP guanyly cyclase receptor of the A subtype; FSK: Forskolin

\section{Acknowledgements}

The authors would like to acknowledge Winson Tay Chung Mun, Subha Subramanian, Shi Yi, Edmund Tan, Hwee Chin Lau and members of Laboratory of Metabolic Medicine at SBIC for help in laboratory work; Krishna $\mathrm{K}$ Chitta for assistance in MATLAB programing for lipid quantification; Brijesh Kumar Singh for assistance in performing the Seahorse XF Cell Mito Stress experiment; Muhammad Shabeer and Asim Shabeer for providing the stromal vascular fractions and maintenance of patient-derived primary cells and Nikon Imaging Centre at Biopolis for support of microscopy usage.

\section{Authors' contributions}

S.C., W.K.O., W.W.Y.Y., W.H., and S.S. designed the experiments. S.C., W.K.O., W.W.Y.Y., Z.Z. performed experiments, data collection, and analysis. B.P.K.N. performed LDs quantification. S.C., S.A.T., W.H., P.M.Y, and S.S. supervised the experiments. S.C. and S.S. drafted the manuscript. S.C., W.K.O., Z.Z., B.P.K.N., S.A.T., W.W.Y.Y., and S.S. revised the article. All authors approved the final version of the manuscript.

\section{Funding}

This work was supported by intramural funding from Biomedical Research Council of A*STAR to S.S.

\section{Availability of data and materials}

The datasets analyzed and materials used during the current study are available either commercially or from the corresponding author upon request.

Ethics approval and consent to participate Use of human cells was conducted with informed consent obtained for each subject, approved by the National Healthcare Group Domain Specific Review Board, Singapore (2009/00376 and 2014/00396), and performed in accordance with its relevant regulations.

Consent for publication Not applicable. 


\section{Competing interests}

S.S. is a co-founder and shareholder of Celligenics Pte. Ltd., which has made no scientific or financial influence over this study. Other authors declare no competing financial interests.

\section{Author details}

${ }^{1}$ Fat Metabolism and Stem Cell Group, Singapore Bioimaging Consortium (SBIC), Agency for Science, Technology and Research (A*STAR) Singapore, 11 Biopolis Way, Singapore 138667, Singapore. ${ }^{2}$ Xenobiology Division, Institute of Bioengineering and Nanotechnology (IBN) Singapore, A*STAR, 31 Biopolis Way, Singapore 138669, Singapore. ${ }^{3}$ School of Pharmacy, Monash University Malaysia, 47500 Selangor, Malaysia. ${ }^{4}$ Cardiovascular and Metabolic Disorders Program, Duke-NUS Medical School Singapore, 8 College Road, Singapore 169857, Singapore. ${ }^{5}$ Signal and Image Processing Group, SBIC, A*STAR Singapore, 11 Biopolis Way, Singapore 138667, Singapore. ${ }^{6}$ Department of Medicine, Yong Loo Lin School of Medicine, National University of Singapore, 5 Lower Kent Ridge Road, Singapore 119074, Singapore. ${ }^{7}$ Laboratory of Metabolic Medicine, SBIC, A*STAR Singapore, 11 Biopolis Way, Singapore 138667, Singapore.

Received: 26 November 2020 Accepted: 20 January 2021

Published online: 04 February 2021

\section{References}

1. Gregor MF, Hotamisligil GS. Inflammatory mechanisms in obesity. Annu Rev Immunol. 2011;29:415-45 Available from: http://www.annualreviews.org/ doi/10.1146/annurev-immunol-031210-101322

2. Deng Y, Scherer PE. Adipokines as novel biomarkers and regulators of the metabolic syndrome. Ann N Y Acad Sci. 2010;1212:E1-19 Available from: http://doi.wiley.com/10.1111/j.1749-6632.2010.05875.x.

3. Cildir G, Akıncılar SC, Tergaonkar V. Chronic adipose tissue inflammation: all immune cells on the stage. Trends Mol Med. 2013;19:487-500 Available from: http://linkinghub.elsevier.com/retrieve/pii/S1471491413000762.

4. Bremer AA, Jialal I. Adipose tissue dysfunction in nascent metabolic syndrome. J Obes. 2013;2013:393192.

5. Zhao M, Chen X. Effect of lipopolysaccharides on adipogenic potential and premature senescence of adipocyte progenitors. Am J Physiol - Endocrinol Metab. 2015;309:E334-44 Available from: http://ajpendo.physiology.org/ lookup/doi/10.1152/ajpendo.00601.2014

6. Ong WK, Tan CS, Chan KL, Goesantoso GG, Chan XHD, Chan E, et al. Identification of specific cell-surface markers of adipose-derived stem cells from subcutaneous and visceral fat depots. Stem Cell Reports. 2014;2:171-9.

7. M. Gimble J, E. Nuttall M. Adipose-derived stromal/stem cells (ASC) in regenerative medicine: pharmaceutical applications. Curr Pharm Des. 2011; 17:332-9. Available from: http://openurl.ingenta.com/content/xref?genre= article\&issn=1381-6128\&volume $=17 \&$ issue $=4 \&$ spage $=332$

8. Lim MH, Ong WK, Sugii S. The current landscape of adipose-derived stem cells in clinical applications. Expert Rev Mol Med. 2014;16:e8.

9. Ong WK, Sugii S. Adipose-derived stem cells: Fatty potentials for therapy. Int J Biochem Cell Biol. 2013:45:1083-6.

10. Wajchenberg BL, Giannella-Neto D, Da Silva MER, Santos RF. Depot-specific hormonal characteristics of subcutaneous and visceral adipose tissue and their relation to the metabolic syndrome. Horm Metab Res. 2002;34:616-21.

11. Després J-P, Lemieux I. Abdominal obesity and metabolic syndrome. Am J Clin Nutr. 2006;79:727-47.

12. Tchkonia T, Tchoukalova YD, Giorgadze N, Pirtskhalava T, Karagiannides I, Forse RA, et al. Abundance of two human preadipocyte subtypes with distinct capacities for replication, adipogenesis, and apoptosis varies among fat depots. Am J Physiol Endocrinol Metab. 2005;288:E267-77.

13. Takeda K, Sriram S, Chan XHD, Ong WK, Yeo CR, Tan B, et al. Retinoic acid mediates visceral-specific adipogenic defects of human adipose-derived stem cells. Diabetes. 2016:65:1164-78.

14. Gunawardana SC, Piston DW. Reversal of type 1 diabetes in mice by brown adipose tissue transplant. Diabetes. 2012;61:674-82.

15. Bartelt A, Bruns OT, Reimer R, Hohenberg H, Ittrich $H$, Peldschus $K$, et al. Brown adipose tissue activity controls triglyceride clearance. Nat Med. 2011; $17: 200-6$.

16. Wang W, Seale P. Control of brown and beige fat development. Nat Rev Mol. Cell Biol. 2016;17:691-702.
17. Wu J, Boström P, Sparks LM, Ye L, Choi JH, Giang AH, et al. Beige adipocytes are a distinct type of thermogenic fat cell in mouse and human. Cell. 2012; 150:366-76

18. Sharp LZ, Shinoda K, Ohno H, Scheel DW, Tomoda E, Ruiz L, et al. Human BAT Possesses Molecular Signatures That Resemble Beige/Brite Cells. PLoS One. 2012;7:e49452.

19. Sugii S, Kida Y, Berggren WT, Evans RM. Feeder-dependent and feederindependent iPS cell derivation from human and mouse adipose stem cells. Nat Protoc. 2011;6:346-58.

20. Yuan C, Chakraborty S, Chitta KK, Subramanian S, Lim TE, Han W, et al. Fast Adipogenesis Tracking System (FATS) - A robust, high-throughput, automation-ready adipogenesis quantification technique. Stem Cell Res Ther. 2019;10:38.

21. Kim JH, Lee SR, Li LH, Park HJ, Park JH, Lee KY, et al. High cleavage efficiency of a 2A peptide derived from porcine teschovirus-1 in human cell lines, zebrafish and mice. PLoS One. 2011;6:e18556.

22. Yau WW, Singh BK, Lesmana R, Zhou J, Sinha RA, Wong KA, et al. Thyroid hormone (T3) stimulates brown adipose tissue activation via mitochondrial biogenesis and MTOR-mediated mitophagy. Autophagy. 2019;15:131-50.

23. Ailhaud G. Cell surface receptors, nuclear receptors and ligands that regulate adipose tissue development. Clin Chim acta. 1999;286:181-90 Available from: http://linkinghub.elsevier.com/retrieve/pii/S000989819900100X.

24. Nofsinger RR, Li P, Hong S-HH, Jonker JW, Barish GD, Ying H, et al. SMRT repression of nuclear receptors controls the adipogenic set point and metabolic homeostasis. Proc Natl Acad Sci U S A. 2008;105:20021-6 Available from: https://doi.org/10.1073/pnas.0811012105\%5Cnhttp://www. pnas.org/content/105/50/20021.full.pdf.

25. Fu M, Sun T, Bookout AL, Downes M, Yu RT, Evans RM, et al. A Nuclear Receptor Atlas: 3T3-L1 Adipogenesis. Mol Endocrinol. 2005;19:2437-50 Available from: https://academic.oup.com/mend/article-lookup/doi/10.1210/ me.2004-0539.

26. Macotela Y, Emanuelli B, Mori MA, Gesta S, Schulz TJ, Tseng YH, et al. Intrinsic differences in adipocyte precursor cells from different white fat depots. Diabetes. 2012;61:1691-9.

27. Denis McGarry J. Dysregulation of fatty acid metabolism in the etiology of type 2 diabetes. Diabetes. 2002;51:7-18

28. Boden $\mathrm{G}$. Role of fatty acids in the pathogenesis of insulin resistance and NIDDM. Diabetes. 1997.

29. Langin D, Dicker A, Tavernier G, Hoffstedt J, Mairal A, Rydén M, et al. Adipocyte lipases and defect of lipolysis in human obesity. Diabetes. 2005; 54:3190-7.

30. Fan XX, Pan HD, Li Y, Guo RJ, Leung ELH, Liu L. Novel therapeutic strategy for cancer and autoimmune conditions: Modulating cell metabolism and redox capacity. Pharmacol. Ther. 2018;191:148-61.

31. Savage DB, Petersen KF, Shulman GI. Disordered lipid metabolism and the pathogenesis of insulin resistance. Physiol Rev. 2007:87:507-20.

32. Choi SM, Tucker DF, Gross DN, Easton RM, DiPilato LM, Dean AS, et al. Insulin regulates adipocyte lipolysis via an Akt-independent signaling pathway. Mol Cell Biol. 2010;30:5009-20.

33. Tran T, Yamamoto Y, Gesta S, Kahn CR. Beneficial effects of subcutaneous fat transplantation on metabolism. Cell Metab. 2008;7:410-20.

34. Tran T, Kahn CR. Transplantation of adipose tissue and stem cells: Role in metabolism and disease. Nat Rev Endocrinol. 2010;6:195-213.

35. Standeven KF, Hess K, Carter AM, Rice Gl, Cordell PA, Balmforth AJ, et al. Neprilysin, obesity and the metabolic syndrome. Int J Obes (Lond). 2011;35: 1031-40 Available from: http://www.pubmedcentral.nih.gov/articlerender. fcgi?artid=3040694\&tool=pmcentrez\&rendertype=abstract.

36. Roques BP, Noble F, Daugé V, Fournié-Zaluski MC, Beaumont A. Neutral endopeptidase 24.11: structure, inhibition, and experimental and clinical pharmacology. Pharmacol Rev. 1993;45:87-146.

37. Maguer-Satta V, Besançon R, Bachelard-Cascales E. Concise review: neutral endopeptidase (CD10): a multifaceted environment actor in stem cells, physiological mechanisms, and cancer. Stem Cells. 2011;29:389-96.

38. Sugii S, Evans RM. Epigenetic codes of PPARy in metabolic disease. FEBS Lett. 2011;585:2121-8.

39. Lefterova MI, Haakonsson AK, Lazar MA, Mandrup S. PPARy and the global map of adipogenesis and beyond. Trends Endocrinol Metab. 2014;25:293302.

40. Langin D, Lucas S, Lafontan M. Millennium fat-cell lipolysis reveals unsuspected novel tracks. Horm Metab Res. 2000;32:443-52. 
41. Schlueter N, De Sterke A, Willmes DM, Spranger J, Jordan J, Birkenfeld AL. Metabolic actions of natriuretic peptides and therapeutic potential in the metabolic syndrome. Pharmacol Ther. 2014;144:12-27.

42. Sengenès $C$, Bouloumié $A$, Hauner $H$, Berlan $M$, Busse R, Lafontan $M$, et al. Involvement of a cGMP-dependent pathway in the natriuretic peptidemediated hormone-sensitive lipase phosphorylation in human adipocytes. J Biol Chem. 2003;278:48617-26.

43. Bezaire V, Mairal A, Ribet C, Lefort C, Girousse A, Jocken J, et al. Contribution of adipose triglyceride lipase and hormone-sensitive lipase to lipolysis in hMADS adipocytes. J Biol Chem. 2009;284:18282-91.

44. Schweiger M, Schreiber R, Haemmerle G, Lass A, Fledelius C, Jacobsen P, et al. Adipose triglyceride lipase and hormone-sensitive lipase are the major enzymes in adipose tissue triacylglycerol catabolism. J Biol Chem. 2006;281: 40236-41.

45. Bolsoni-Lopes A, Alonso-Vale MIC. Lipolysis and lipases in white adipose tissue - An update. Arch. Endocrinol Metab. 2015;59:335-42.

46. Holm C. Molecular mechanisms regulating hormone-sensitive lipase and lipolysis. Biochem Soc Trans. 2003;31:1120-4.

47. Su CL, Sztalryd C, Contreras JA, Holm C, Kimmel AR, Londos C. Mutational analysis of the hormone-sensitive lipase translocation reaction in adipocytes. J Biol Chem. 2003;278:43615-9.

48. Steinberg GR, Kemp BE, Watt MJ. Adipocyte triglyceride lipase expression in human obesity. Am J Physiol - Endocrinol Metab. 2007;293:E958-64.

49. Jocken JWE, Langin D, Smit E, Saris WHM, Valle C, Hul GB, et al. Adipose triglyceride lipase and hormone-sensitive lipase protein expression is decreased in the obese insulin-resistant state. J Clin Endocrinol Metab. 2007; 92:2292-9.

50. Large V, Reynisdottir S, Langin D, Fredby K, Klannemark M, Holm C, et al. Decreased expression and function of adipocyte hormone-sensitive lipase in subcutaneous fat cells of obese subjects. J Lipid Res. 1999;40:2059-65.

51. Rodriguez-Cuenca S, Carobbio S, Velagapudi VR, Barbarroja N, MorenoNavarrete JM, Tinahones FJ, et al. Peroxisome proliferator-activated receptor $\gamma$-dependent regulation of lipolytic nodes and metabolic flexibility. Mol Cell Biol. 2012;32:1555-65.

52. Kershaw EE, Schupp M, Guan HP, Gardner NP, Lazar MA, Flier JS. PPARY regulates adipose triglyceride lipase in adipocytes in vitro and in vivo. Am J Physiol - Endocrinol Metab. 2007:293:E1736-45.

53. Tamori Y, Masugi J, Nishino N, Kasuga M. Role of peroxisome proliferatoractivated receptor- $\gamma$ in maintenance of the characteristics of mature 3T3-L1 adipocytes. Diabetes. 2002;51:2045-55.

54. Kim SH, Plutzky J. Brown fat and browning for the treatment of obesity and related metabolic disorders. Diabetes Metab J. 2016;40:12 Available from: https://synapse.koreamed.org/DOlx.php?id=10.4093/dmj.2016.40.1.12.

55. Ye L, Kleiner S, Wu J, Sah R, Gupta RK, Banks AS, et al. TRPV4 is a regulator of adipose oxidative metabolism, inflammation, and energy homeostasis. Cell. 2012;151:96-110 Available from: http://linkinghub.elsevier.com/retrieve/ pii/S0092867412011026.

56. Seale P, Conroe HM, Estall J, Kajimura S, Frontini A, Ishibashi J, et al. Prdm16 determines the thermogenic program of subcutaneous white adipose tissue in mice. J Clin Invest. 2011:121:96-105.

57. Kiefer FW, Vernochet C, O'Brien P, Spoerl S, Brown JD, Nallamshetty S, et al. Retinaldehyde dehydrogenase 1 regulates a thermogenic program in white adipose tissue. Nat Med. 2012;18:918-25.

58. Vegiopoulos A, Müller-Decker K, Strzoda D, Schmitt I, Chichelnitskiy E, Ostertag A, et al. Cyclooxygenase-2 controls energy homeostasis in mice by de novo recruitment of brown adipocytes. Science (80- ). 2010;328:1158-61.

\section{Publisher's Note}

Springer Nature remains neutral with regard to jurisdictional claims in published maps and institutional affiliations.

Ready to submit your research? Choose BMC and benefit from:

- fast, convenient online submission

- thorough peer review by experienced researchers in your field

- rapid publication on acceptance

- support for research data, including large and complex data types

- gold Open Access which fosters wider collaboration and increased citations

- maximum visibility for your research: over $100 \mathrm{M}$ website views per year

At BMC, research is always in progress.

Learn more biomedcentral.com/submissions 